\title{
Medición de la capacidad de carga turística de Cozumel
}

\author{
Romano Segrado \\ Alejandro Palafox Muñoz / Lucinda Arroyo
}

RESUMEN

El estudio inició como una iniciativa planeada de reducción de los impactos negativos por la presencia de visitantes en un destino masivo y también zona protegida. Como primera parte de este proceso, la determinación de la capacidad de carga que representa una aproximación estimada de visitantes que un destino turístico puede aceptar sin producir daños serios al sistema ambiental. La estimación puede ser hecha por varios métodos, pero en esta investigación fue aplicado el método de Cifuentes (1999) y el análisis de indicadores claves, además del uso del GPS, en la isla de Cozumel, México.

El objetivo primario fue determinar los indicadores como estrategia para gestionar la investigación, y como resultado, definir un valor recomendable de visitantes que el destino debería recibir diariamente.

La investigación fue financiada por la Universidad de Quintana Roo.

\section{PALABRAS CLAVE}

Turismo, Capacidad de carga. Metodología. Indicadores. 


\title{
Measurement of the touristic carrying capacity in Cozumel
}

\author{
Romano Segrado \\ Alejandro Palafox Muñoz / Lucinda Arroyo
}

\begin{abstract}
SUMMARY
This study began as an planned initiative of reduction of the negative impacts caused by the presence of visitors in a massive destination and protective zone, too. As a first part, this process determines the capacity of load of an estimated approximation of visitors that a destination can accept without producing serious damage to the environmental system. Such estimation can be done by several methods, but this research uses the method of Cifuentes (1999) and the analysis of key indicators, apart from the use of GPS in the island of Cozumel, Mexico.
\end{abstract}

The prime objective was to determine the indicators as strategy to negotiate the research, and as a result, to define recommendable value of visitors that the destination should receive daily.

The research was sponsored by Universidad de Quintana Roo.

\section{KEY WORDS}

Tourism, carrying capacity, methodology, indicators. 


\section{INTRODUCCIÓN}

El propio plan de desarrollo de Quintana Roo 2005-2011 reconoce que es necesario revisar el modelo actual de desarrollo turístico, tanto a nivel estatal como a nivel de los destinos individuales dentro del Estado, para que los mismos se adecuen a la normatividad nacional e internacional sobre sustentabilidad' ambiental de destinos turísticos, que implica directamente lograr beneficios concretos y permanentes, en armonía con el medio natural y social, paras las comunidades receptoras de la actividad turística.

Lograr estos beneficios y compatibilizarlos con la sustentabilidad ambiental no es tarea fácil, ya que los factores que deben combinarse muchas veces no son complementarios sino antagónicos -intereses sociales y económicos, unos a corto plazo y otros a largo plazo- y también por el hecho de que la combinación de recursos necesarios para lograr la sustentabilidad ambiental requiere la determinación de prioridades para la solución de problemas que históricamente han sido postergados, planteándose la necesidad de elegir entre problemas urgentes, importantes o impostergables.

\section{Romano Segrado}

Maestría en Estudios Turísticos UAEM

Linea de investigación: Capacidad de Carga Turística, Fenómenos naturales y turismo

Coordinador de la Lic. en Turismo

Líder del Cuerpo Académico de Turismo (CAT)
Sin embargo, aún con todos estos inconvenientes, el turismo es una opción válida como impulsor del crecimiento económico y de un polo de desarrollo, pero este mismo motivo exige que esta actividad se dirija hacia la sustentabilidad, ya que la sociedad no sólo necesita asegurar su crecimiento y bienestar para los próximos 20 ó 25 años, sino por todo su devenir histórico, que es la visión a futuro que se desea para la entidad.

El Plan Estratégico de Desarrollo Integral del Estado de Quintana Roo 20002025 PEDIEQRoo (2000: 145), destaca al turismo como "el principal motor de su desarrollo económico, estableciendo en el estado una dinámica permanente que integre al resto de los sectores de la economía, generando oportunidades de empleo y desarrollo tanto económico como social para sus habitantes en 
medio de un clima de oportunidades empresariales con un marco de libre competencia", complementado con la sustentabilidad económica, social y natural.

Sin embargo, a pesar de una visión ideal y unas ambiciosas, en la práctica la política turística estatal tiene un objetivo de corto plazo que busca contribuir a la solución de problemas económicos y sociales propios de un país en vías de desarrollo, por medio de la captación de divisas y la generación de empleos, y a largo plazo obtener una posición estratégica como destino turístico de los EUA, a la vez de convertirse en el eje del desarrollo estatal. Esta política implica de facto hacer caso omiso o, muy reducido, a las políticas nacionales o estatales referentes a la sustentabilidad.

Por supuesto, la situación ideal sería que la actividad turística fomente un desarrollo sustentable, aunque actualmente se sigue promoviendo el mismo modelo turístico de los años cincuenta: playa y sol, continuando con la política de masificación, que si bien no da señales de agotamiento, sino de un crecimiento sostenido, determina una especialización y dependencia económica muy alta y que dista bastante de ser sustentable a corto, mediano o largo plazo.

Con esta afirmación no se sugiere cambiar abruptamente la política turística estatal, sino recomendar acciones que impulsen la sustentabilidad de los destinos turísticos del Estado de Quintana Roo.
Alejandro Palafox

Muñoz

Maestría en Estudios

Turísticos.

Secretario Técnico de Posgrado e Investigación de la División de Desarrollo Sustentable en la Universidad de Quintana Roo

Editor de la revista Teoría y Praxis

\section{Lucinda Arroyo}

Maestría en Economía Y Administración Pública, Universidad de Quintana Roo

Estudios de Maestría en Ciencias de la Planificación y Desarrollo Regional. Instituto Tecnológico de Mérida

Jefe de Departamento de Estudios Sociales y Empresariales, Universidad de Quintana Roo, . Unidad Académica Cozumel.

Participación en proyectos de investigación internos $y$ externos 


\section{EL DESTINO TURÍSTICO}

La isla de Cozumel se encuentra localizada en el suroeste de la República Mexicana, en el Municipio de Cozumel (Figura 1), y cuenta con una superficie de 48,129 hectáreas, en donde el 75\% está cubierta por una vegetación consistente en selva mediana sub-perenifolia con asociaciones de manglar en zonas inundables y la línea de costa (Periódico Oficial del Gobierno del Estado de Quintana Roo POGEQR 21/05/2002).

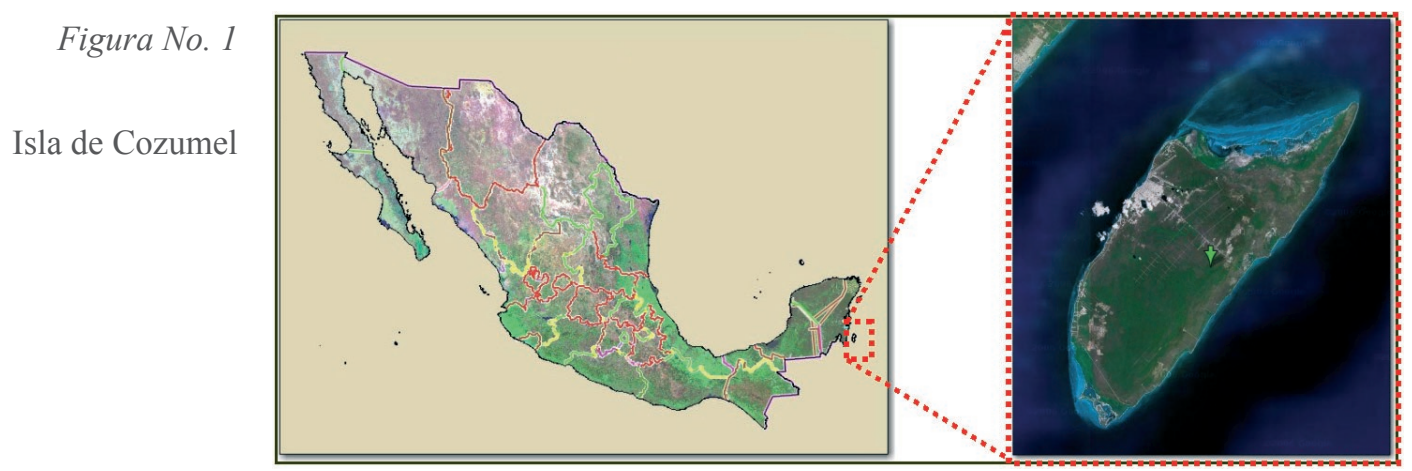

Fuente: INEGI, 2007; Google Earth 2007.

La isla cuenta con varios ecosistemas de gran belleza y una ubicación privilegiada en el Caribe Mexicano, presentando condiciones favorables para el desarrollo de la actividad turística, la cual se manifiesta con el gran afluente de cruceros turísticos a nivel mundial que arriban a ella. Tiene dos áreas naturales protegidas, una de competencia federal, y la otra de competencia estatal (Periódico Oficial del Gobierno del Estado de Quintana Roo POGEQR 21/05/2002).

Con respecto a la comunidad local, el modelo turístico en la región ha producido un nuevo orden territorial caracterizado por un proceso de concentración urbana. El 99\% de la población se localiza en el puerto-ciudad de San Miguel, con una tasa de crecimiento de 7.1\% anual. La migración, atraída por el desarrollo de la actividad terciaria ha tenido como consecuencia una constante demanda de suelo urbano y un aumento en la mancha urbana de 38 hectáreas por año (HAC Plan de Desarrollo Municipal Cozumel PDMC 2005-2008, 2005). 
Paralelamente a esta migración y urbanización, los espacios naturales se han popularizado para el uso turístico y recreativo, lo cual ha conllevado a su masificación. Esta problemática se agrava por el hecho que no existe gestión alguna del patrimonio y recurso natural. Por otra parte la creciente capacidad de movilidad interna que han generado los nuevos hábitos turísticos, especialmente con el incremento de los excursionistas ${ }^{2}$ (visitantes de cruceros) (Tabla 1) y del consecuente alquiler de vehículos, ha provocado que muchas barreras naturales hayan cedido ante la presión del turismo, provocando situaciones de masificación en espacios antes inexplorados.

\begin{tabular}{|c|c|c|c|}
\hline Año & Cruceristas & Transbordador & Turistas \\
\hline 1996 & 985,656 & ND & ND \\
\hline 1997 & $1,087,882$ & ND & ND \\
\hline 1998 & $1,142,619$ & ND & 416,838 \\
\hline 1999 & $1,341,203$ & ND & 398,737 \\
\hline 2000 & $1,504,604$ & $1,153,476$ & 421,541 \\
\hline 2001 & $1,567,614$ & $1,150,636$ & 455,620 \\
\hline 2002 & $2,338,781$ & $1,130,844$ & 383,676 \\
\hline 2003 & $2,708,913$ & $1,296,851$ & 364,787 \\
\hline 2004 & $2,864,137$ & $1,422,654$ & 418,598 \\
\hline 2005 & $2,519,179$ & $1,374,145$ & 396,734 \\
\hline 2006 & $2,350,838$ & $1,256,653$ & ND \\
\hline
\end{tabular}

Fuente: Elaboración propia con datos de DATATUR, API, SCT, SEDETUR.

Estos impactos han sido identificados por el propio Plan de Desarrollo Municipal de Cozumel (HAC Plan de Desarrollo Municipal Cozumel 2005-2008, 2005), donde se afirma que los problemas turísticos "se han agudizado por (...) la consolidación y aparición de nuevos destinos turísticos, los cambios en los hábitos de consumo y satisfactores de los turistas, deterioro en la calidad de desarrollo, presiones por el acelerado crecimiento urbano y sus impactos sobre los entornos naturales" (HAC Plan de Desarrollo Municipal Cozumel 2005-2008). 
Esta paradoja del sector turístico es debida a sus relaciones con el medio natural, ya que por un lado consume recursos naturales y por el otro necesita un entorno natural atractivo para su desarrollo. Sin embargo, correctamente planificado en su gestión y con programas de conservación del ambiente es posible evitar los impactos negativos y potenciar los positivos, aunque para lograr esto es necesario el conocimiento de los patrones de uso sobre dichos espacios, que es un aspecto primordial para gestionar satisfactoriamente la demanda social a la que están sometidos, y para evitar o aliviar los impactos naturales y sociales. 


\section{LA ACTIVIDAD TURÍSTICA}

Como característica principal de la actividad turística en Cozumel, se hace uso intensivo de los recursos naturales, los cuales se complementan con la oferta cultural (la zona arqueológica de San Gervasio, el Museo de la isla, el Carnaval Cozumeleño, el Cedral, el Caracol, por citar a los más importantes), sin embargo el desarrollo turístico de Cozumel se ha realizado sin la protección adecuada del ambiente, que es un requisito esencial de su propio desarrollo, ya que sin una adecuada calidad ambiental el turismo actual y futuro pueden verse comprometidos, (Cater, citado por Santana, 2003) por el doble papel que el turismo implica; por un lado impulsor del crecimiento socioeconómico y por el otro destructor del medio natural y social.

Como impactos principales, la tabla 2 presenta los aspectos positivos y negativos naturales y sociales del desarrollo turístico y recreativo de Cozumel.

\begin{tabular}{|c|c|c|c|c|}
\hline Ecológicos & Económicos & Sociales & Estéticos & \multirow{8}{*}{$\begin{array}{l}\text { Impactos } \\
\text { causados por } \\
\text { el turismo en } \\
\text { Cozumel }\end{array}$} \\
\hline $\begin{array}{l}\text { Destrucción de } \\
\text { arrecifes }\end{array}$ & $\begin{array}{l}\text { Aumento del costo de } \\
\text { limpieza urbana }\end{array}$ & $\begin{array}{l}\text { Descomposición de las } \\
\text { redes sociales }\end{array}$ & $\begin{array}{l}\text { Destrucción de la } \\
\text { fisonomía urbana }\end{array}$ & \\
\hline $\begin{array}{l}\text { Degradación } \\
\text { de habitats }\end{array}$ & $\begin{array}{l}\text { Aumento de valor de la } \\
\text { tierra y su uso }\end{array}$ & $\begin{array}{l}\text { Exclusión de la } \\
\text { comunidad en nuevas } \\
\text { empresas turísticas }\end{array}$ & $\begin{array}{l}\text { Pérdida de espacios } \\
\text { recreativos } \\
\text { comunales }\end{array}$ & \\
\hline $\begin{array}{l}\text { Alta demanda } \\
\text { de agua dulce }\end{array}$ & $\begin{array}{l}\text { Sobrecarga de } \\
\text { infraestructura }\end{array}$ & $\begin{array}{l}\text { Cambios en los } \\
\text { horarios laborales }\end{array}$ & $\begin{array}{l}\text { Aglomeración de } \\
\text { personas }\end{array}$ & \\
\hline $\begin{array}{l}\text { Aumento de la } \\
\text { población }\end{array}$ & $\begin{array}{l}\text { Pérdida de capacidad } \\
\text { productiva en oferta no } \\
\text { turística }\end{array}$ & Conflictos culturales & $\begin{array}{l}\text { Congestionamiento } \\
\text { del tránsito }\end{array}$ & \\
\hline $\begin{array}{l}\text { Importación de } \\
\text { flora y fauna no } \\
\text { autóctona }\end{array}$ & $\begin{array}{l}\text { Generación de } \\
\text { empleos directos e } \\
\text { indirectos }\end{array}$ & $\begin{array}{l}\text { Migración (efecto } \\
\text { simultáneo positivo y } \\
\text { negativo) }\end{array}$ & & \\
\hline \multirow[t]{2}{*}{$\begin{array}{l}\text { Pérdida de } \\
\text { biodiversidad }\end{array}$} & $\begin{array}{l}\text { Creación de pequeñas } \\
\text { empresas }\end{array}$ & $\begin{array}{l}\text { Segregación } \\
\text { residencial }\end{array}$ & & \\
\hline & $\begin{array}{l}\text { Fomento de la mano } \\
\text { de obra no capacitada }\end{array}$ & $\begin{array}{l}\text { Brechas sociales } \\
\text { (extranjeros ocupan } \\
\text { posiciones superiores) }\end{array}$ & & \\
\hline
\end{tabular}

Fuente: Observación de campo, 2007.

Un aspecto fundamental para evitar o aliviar estos impactos turísticos es el flujo de visitantes, ya que estos ejercen una presión que es considerada como significante a nivel de la isla, aunque 
aún no se cuenta con información técnico-científica necesaria para determinar el nivel actual de tolerancia de la isla, con respecto a visitantes y turistas, lo que en términos técnicos se denomina como Capacidad de Carga Turística ${ }^{3}$ (CCT).

Los parques naturales, las playas, y los espacios costeros de la isla de Cozumel constituyen un importante recurso turístico y recreativo. La presión turística y recreativa sobre ellos, en especial la concentrada durante ciertos periodos del año, principalmente desde Octubre a Febrero (Figura 2), genera impactos naturales y sociales de diversa índole, algunos importantes y urgentes, y otros no tanto. Todos ellos son inmensurables, derivados de la frecuentación de excursionistas y turistas (principalmente los primeros), además de los propios habitantes de la isla, quienes generan importantes cantidades de residuos sólidos, fragmentación de hábitats naturales, y desencadenamiento de procesos erosivos, entre otros.

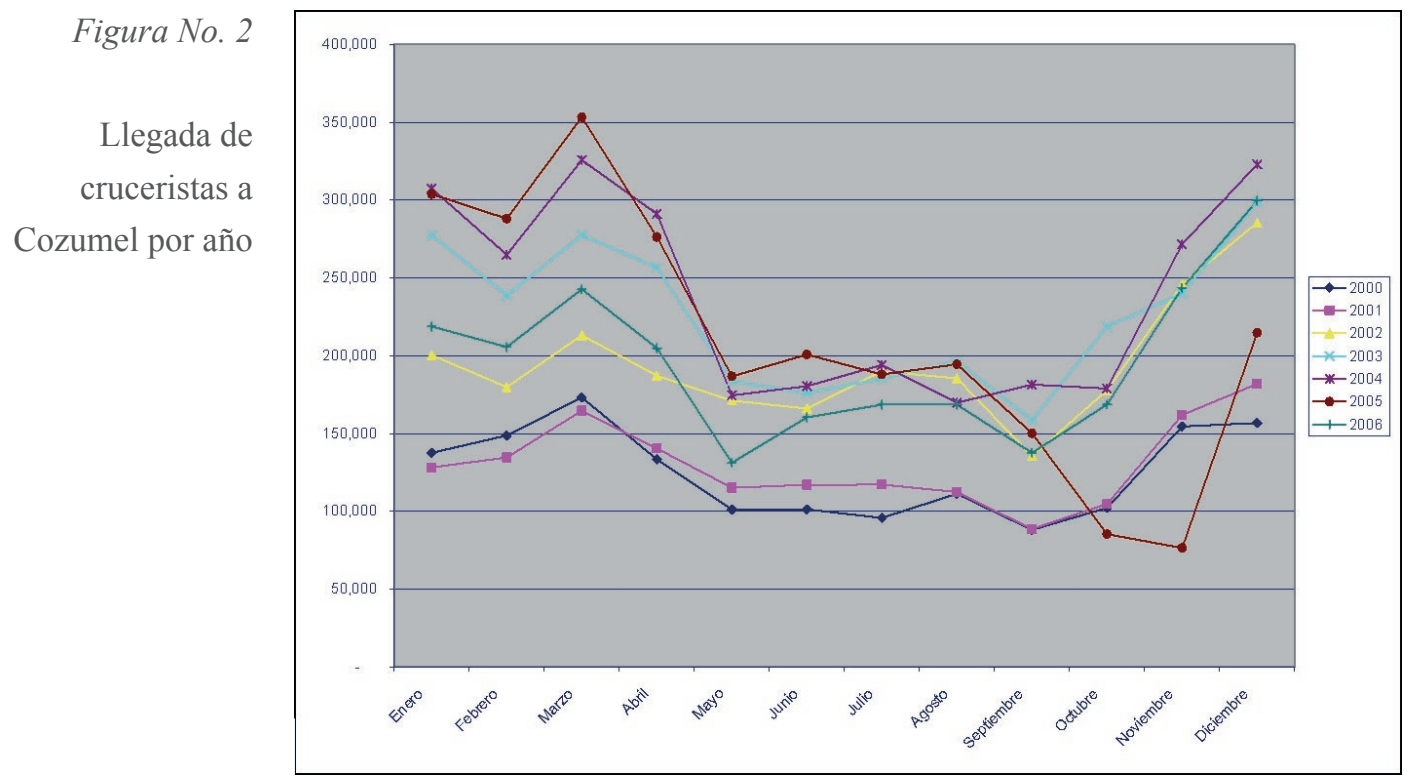

Fuente: Elaboración propia con datos de DATATUR, API, SCT, SEDETUR. 
La mejor forma de evitar y prevenir estas afectaciones es por medio del conocimiento de este límite natural y social, con un indicador de desarrollo turístico sustentable viable y aceptado a nivel internacional, tal como lo es la CCT: una evaluación imprescindible para realizar programas y propuestas de gestión que apoyen y complementen el Plan de Ordenamiento Ecológico Territorial (POET) de Cozumel.

La capacidad de carga es una estrategia potencial ${ }^{4}$ para reducir los impactos de la recreación de los visitantes en los destinos turísticos y recreativos (Cifuentes et al., 1999). Por otra parte, un estudio de CCT de Cozumel es un instrumento eficaz para orientar la protección del ambiente y la conservación y utilización adecuada de los recursos naturales, con base en criterios e instrumentos, desde una perspectiva integradora y preventiva, fomentando el desarrollo de las acciones de manejo y aprovechamiento de los ecosistemas, tal como se ha declarado en el Plan Básico de Gobierno 1999- 2005 del Estado de Quintana Roo, al que se la ha dado continuidad en el Plan de Gobierno 2006-2011.

En Cozumel, la actividad económica desarrollada está basada principalmente en los $\operatorname{cruceros}^{5}$ y en menor grado en las segundas residencias (Observación de campo, 2007), por lo que representa un modelo turístico con escaso potencial socioeconómico y con una gran depredación de recursos naturales, además de crear una dependencia general hacia la actividad (Tabla 3).

Por otra parte, el segmento mayoritario de los excursionistas y o turistas que llegan a Cozumel tienen base en elementos externos al sistema económico y social nacional (turistas y excursionistas norteamericanos, canadienses y europeos) y no tiene el nivel deseado de educación ambiental y esto contribuye a potenciar aún más la ya clásica "tragedia de los recursos comunes", lo cual indica que es lógico fijar un límite máximo o, cuando menos, conocer qué tan lejos se está de la situación idónea, logrando cierto control sobre la actividad turística y el desarrollo sustentable de la isla. 


\begin{tabular}{|c|c|c|c|}
\hline Tabla No. 3 & Categoría/Tipo & Turismo masivo & $\begin{array}{c}\text { Turismo } \\
\text { alternativo }\end{array}$ \\
\hline \multirow{6}{*}{$\begin{array}{r}\text { Tipología de } \\
\text { la actividad } \\
\text { turística }\end{array}$} & Producto & Paquete turístico & Ecoturismo \\
\hline & Actividad & Pasiva & Activa \\
\hline & Preferencia & Playa & Naturaleza \\
\hline & Duración & Horas & Días \\
\hline & Motivo & Recreación & Cultura \\
\hline & Poder adquisitivo & Bajo a medio & Medio a superior \\
\hline
\end{tabular}

Fuente: Observación de campo, 2007; SEDETUR 2005.

En el municipio ya existen algunos estudios de capacidad de carga turística para los parques naturales Chankanaab y Punta Sur, para el área terrestre, no así para el ámbito acuático, y lo que se pretende aquí es utilizar los conceptos metodológicos de Cifuentes (1999) y proponer una metodología con un enfoque más amplio, que abarque toda la isla en su conjunto, en el sector terrestre. 


\section{LA CAPACIDAD DE CARGA TURÍSTICA}

Históricamente, los esfuerzos por lograr un enfoque unificado al impacto de la actividad turística en los destinos han sido débiles y parciales, debido a la fundamentación descriptiva, que no proporciona un marco para el análisis (Cooper; Fletcher; Gilbert; Wanhill, 1997), aunque se puede inferir que los diversos métodos actuales se apropian del concepto de desarrollo sustentable "con la pretensión de ser más globalizadoras y comprehensivas" (García, 2003: 72), bajo el concepto de que la protección de los recursos turísticos es el presupuesto básico para una actividad turística perdurable.

Sobre el turismo sustentable o sostenible, la Organización Mundial del Turismo (OMT, 1993) lo define como aquel turismo que "atiende a las necesidades de los turistas actuales y de las regiones receptoras $\mathbf{y}$ al mismo tiempo protege $\mathbf{y}$ fomenta las oportunidades para el futuro. Se concibe como una vía hacia la gestión de todos los recursos de forma que puedan satisfacer las necesidades económicas, sociales y estéticas, respetando al mismo tiempo la integridad cultural, los procesos ecológicos esenciales, la diversidad biológica y los sistemas que sostiene la vida" (citado por la Fundación para el Desarrollo Sustentable, s.f.).

Esta definición pone énfasis en el equilibrio entre los aspectos naturales, sociales y económicos del turismo, así como en la necesidad de aplicar principios de sustentabilidad en todos los sectores de la actividad turística, como por ejemplo cuando no se sobrepasa la capacidad de carga (CCT) de un destino turístico (WIKI, 2007), por lo tanto se puede hablar de turismo sustentable. Su determinación constituye una herramienta de planificación que permite obtener una aproximación a la intensidad de uso de las áreas destinadas al uso público por lo que sustenta y requiere decisiones de manejo (Cifuentes, 1999).

El cálculo se hace mediante un proceso complejo en el que se deben considerar una serie de indicadores ecológicos, físicos, sociales, económicos y culturales. Sin embargo, aunque el postulado teórico es casi ideal, el problema se encuentra en el momento de establecer los indicadores que determinarán la capacidad de carga máxima del destino, ya que los mismos deben definirse en función de cada destino turístico. 
Para establecer los indicadores de la CCT fue seleccionado el método de Cifuentes (1999), adaptado a las características propias de esta región, debido a que se detectó una ausencia de métodos específicos para la operacionalización de la CCT, ya que si bien existe amplia documentación teórica, se aprecia una carencia importante en cuanto a las aplicaciones para los destinos turísticos ${ }^{7}$, más aún en destinos de islas o destinos de cruceros, que considere tanto visitantes como turistas, debido a los parámetros, variables, y complejidad de relaciones que se presentan en los espacios urbanos (García, 2003).

Un último motivo por el cual se consideró conveniente aplicar el modelo de Cifuentes (1999) fue la consideración sobre los modelos de evolución de los destinos turísticos de Cohen (1972), Butler (1980), y Smith (1989), que sugieren que los destinos turísticos maduros deben aplicar técnicas directas (Ffolliott et al., 2001) (Tabla 4) de planificación turística para aquellos destinos en que se ofertan paquetes turísticos donde hay poco contacto con la población local, tal como lo son los flujos continuos de cruceros y los charters. 


\begin{tabular}{|c|c|}
\hline Método & Técnica \\
\hline \multirow{4}{*}{$\begin{array}{l}\text { Ampliación de las } \\
\text { regulaciones }\end{array}$} & Incremento del patrullaje \\
\hline & Imposición de tasas \\
\hline & Acompañamiento obligatorio de guía \\
\hline & Uso de cámaras de vigilancia \\
\hline \multirow[t]{2}{*}{ Zonificación } & $\begin{array}{l}\text { Prohibición de ciertos tipos de usos en } \\
\text { ciertas zonas (e.g.| botes sobre } \\
\text { arrecifes) }\end{array}$ \\
\hline & Cerrar campamentos \\
\hline \multirow{3}{*}{ Ratio de uso } & $\begin{array}{l}\text { Limitar el tamaño de los grupos de } \\
\text { visitantes }\end{array}$ \\
\hline & Limitar el tiempo de estadía \\
\hline & $\begin{array}{l}\text { Limitar el total de personas que } \\
\text { pueden acceder al destino }\end{array}$ \\
\hline \multirow{5}{*}{$\begin{array}{l}\text { Restricción de } \\
\text { actividades }\end{array}$} & Prohibir fogatas \\
\hline & Prohibir campamentos \\
\hline & $\begin{array}{l}\text { Prohibir el acceso con mascotas o } \\
\text { animales que puedan causar } \\
\text { alteraciones }\end{array}$ \\
\hline & Prohibir el abandono de basuras \\
\hline & $\begin{array}{l}\text { Prohibir el pastaje de animales } \\
\text { (caballos, vacas, etc.) }\end{array}$ \\
\hline
\end{tabular}

Tabla No. 4

Técnicas

Directas

Fuente: Ffolliott et al., 2001

El método de Cifuentes (1999) tiene varios beneficios, tales como: el procedimiento al aplicarlo es compresible y sencillo para determinar la capacidad de carga del área en estudio. La metodología también se apoyó en Navarro (2005), quien sugiere una serie de indicadores (alojamiento, vialidad, abastecimiento y saneamiento de agua) para la evaluación de la CCT, para destinos turísticos costeros. 
Con el método Cifuentes (1999), se pretende establecer el número máximo de visitas que puede recibir un área determinada, con base en las condiciones físicas, biológicas y de manejo que se presentan en el área en el momento del estudio (estudio transversal). El proceso consta de tres niveles:

1. Cálculo de Capacidad de Carga Física (CCF)

2. Cálculo de Capacidad de Carga Real (CCR)

3. Cálculo de Capacidad de Carga Efectiva (CCE), donde la CCF > CCR > CCE.

Como parte del estudio de Carga Física, se realizó la caracterización temporal de los visitantes, con su estancia media, ya sea en horas o días. Adicionalmente, fue necesario determinar la ubicación espacial de los atractivos de acuerdo a su situación geográfica, y para ello se utilizó el sistema geoposicional GPS (Geographyc Positional System), para determinar y analizar las áreas geográficas de mayor presencia o concentración turística, ya que no sólo se debe considerar el factor demográfico sino también las formas de apropiación y la intensidad de uso del destino o sitios turísticos (Wurz, 2003). Adicionalmente, la herramienta GPS ${ }^{8}$ permite asociar ciertos datos con su ubicación geográfica, como el ejemplo de la tabla 5 (geo-referenciación) (Star y Estes, 1990).

Con la información geo-referenciada se pueden construir mapas para investigar la naturaleza y tendencias espaciales de variables de interés (por ejemplo, número de habitaciones y camas). Con los SIG, es además posible realizar operaciones espaciales que permiten asociar múltiples bases de datos con relación a su posición geográfica y que de otra forma no podrían combinarse (número de hoteles en un área determinada alrededor de un arrecife, como en el caso de esta investigación).

La utilización de estos sistemas en el análisis espacial de los niveles de cobertura, accesibilidad y utilización de servicios, puede servir como un importante insumo en la evaluación de la capacidad de carga turística. 


\begin{tabular}{|c|c|c|c|}
\hline SITIO & Sistema de Proyección & Q & UTM \\
\hline UQROO & UTM Zona 16 (Datum & 0506071 (metros) & 2265533 (metros) \\
Cozumel & horizontal: WGS84 & & \\
\hline
\end{tabular}

Tabla No. 5 COORDENADAS

Fuente: Elaboración propia. (formato $\mathrm{X}-\mathrm{Y}$ )

Debido a que el estudio se realizó en la isla de Cozumel, fue necesario realizar un inventario de todos los atractivos turísticos y de las ofertas de servicios turísticos y recreación existentes en la isla, junto con su infraestructura, capacidad de alojamiento de personas y otros factores importantes. Para llevar a cabo dicho inventario se utilizó el Tourism Rapid Assessment Tool (TRAT), instrumento recomendado por la Organización Conservation International y la George Washington University. 


\section{LOS INDICADORES}

"Para examinar el impacto del turismo de manera objetiva, es necesario hacer a un lado los conceptos de contaminación de aguas, congestión de tráfico y playas saturadas que conllevan juicios de valor" (Cooper; Fletcher; Gilbert; Wanhill, 1997: 112), y bajo la lógica de que es necesario utilizar instrumentos críticos para el análisis de la actividad humana, se determinó el uso de indicadores por los siguientes motivos: Relevancia (significación para el tomador de decisiones), Credibilidad (verosimilidad del indicador), Legitimidad (sesgo hacia los intereses de alguien), Factibilidad (posibilidad de construir el indicador dependiendo de la disponibilidad de datos, costos, etc.) (Gallopín, 2006).

Los indicadores fueron diseñados con fines evaluadores y no descriptivos, con el fin de captar la complejidad de la actividad turística y presentarla en términos simples, para luego ir generando un indicador compuesto, como es la CCT. Para la determinación de la CCT de Cozumel, fueron elaborados 24 indicadores, creados individualmente a partir de una "ficha técnica de indicador" (ver Apéndice 1), donde se estableció la clave, nombre, descripción, objetivo, definición operativa, importancia, comentarios, fuente de información, fórmula, variables, y unidades de medida.

En la primera fase, se determinó la Capacidad de Carga Física ${ }^{9}$ (CCF), que es el límite máximo de visitas que se pueden hacer al sitio durante un día. Es la resultante de la relación entre factores de visita (horario y tiempo de visita), el espacio disponible y la necesidad de espacio por visitante. Para el cálculo se elaboraron indicadores, con la fórmula recomendada por Cifuentes (1999):

- Indicador de Superficie turística disponible (S),

- Indicador de Superficie turística usada por persona (SP),

- Indicador de Total de horas de atención a visitantes (HV),

- Indicador de Tiempo necesario para la visita (TN).

En la segunda fase, se sometió la CCF a una serie de factores de corrección, particulares para cada sitio, siguiendo el método de Cifuentes (1999) y según lo señalado por Mitraud (1997: sd.), quien afirma que cualquier metodología que pretenda determinar la capacidad de carga debe considerar lo siguiente: "El manejo de la visitación no exige una cantidad pre-establecida de información 
disponible para ser analizada. Esto porque tanto ahora como a futuro, debe aceptarse que se desconoce más de lo que se conoce sobre las complejas e innumerables interrelaciones de un ecosistema".

Los indicadores y factores de corrección fueron:

- Indicador de Superficie turística usada por ciudadanos (FCesp)

- Indicador de Tiempo de espera para acceder a facilidades (FCero)

- Indicador de Disponibilidad de agua potable por visitante por día (FCagu)

- Indicador de Disponibilidad de electricidad por visitante por día (FCene)

- Indicador de Intensidad del tránsito (FCtra)

- Indicador de Precipitación (FCpre)

- Indicador de Cierres temporales (FCtem)

- Indicador de Superficie turística degradada (FCdeg)

Estos factores se calcularon en función de una fórmula general que considera la magnitud total de cada variable " $x$ " y la magnitud limitante de la misma variable " $x$ ", considerando el valor total disponible, el valor utilizado por la población local y el valor remanente disponible para los visitantes.

$\mathrm{FCx}=\frac{\mathrm{Mlx}}{\mathrm{Mtx}}$

donde:

FCx = Factor de corrección por la variable " $\mathrm{x"}$

$M l x=$ Magnitud limitante de la variable " $x$ "

Mtx = Magnitud total de la variable "x" (Cifuentes, 1999).

En la tercera fase, se determinó la Capacidad de Manejo (CM), donde intervienen variables como respaldo jurídico, políticas, equipamiento, dotación de personal, financiamiento, infraestructura y facilidades o instalaciones disponibles (Cifuentes, 1999). La capacidad de manejo óptima es 
definida como el mejor estado o condiciones que la administración (o para este caso particular el municipio) debe tener para desarrollar sus actividades y alcanzar sus objetivos.

Los indicadores fueron seleccionados porque son factores limitantes de la actividad turística, también por la facilidad de análisis, y debido a que se contó con la información requerida para cada indicador, además de que varios de ellos permiten medir el nivel de sustentabilidad del destino, en sus puntos críticos, según dos de los cuatro principios de sustentabilidad de Daly (1994):

1. No explotar los recursos renovables por encima de su tasa de renovación;

2. No verter residuos al ambiente por encima de su capacidad de asimilación.

En la última fase, la Capacidad de Carga Efectiva (CCE) representa el número máximo de visitas (por excursionistas y turistas) que se puede permitir en la isla de Cozumel. La CCE se determinó con esta fórmula: $\mathrm{CCE}=\mathrm{CCR} \times \mathrm{CM}$. 


\section{REFLEXIONES FINALES}

El resultado principal fue la demostración de que la metodología de Cifuentes (1999), con las modificaciones realizadas, es un instrumento analítico válido para determinar la Capacidad de Carga Turística (CCT) en Cozumel o en otros municipios del Estado. Quintana Roo, además de alertar sobre los factores que limitan el crecimiento del turismo, ya que el concepto de CCT no debe ser interpretado exclusivamente como limitador, sino también como identificador de los factores vinculados al crecimiento de la actividad turística.

Además de esto, con la construcción de la CCT por medio de indicadores mediante el uso de fichas técnicas, se eliminó el problema de los juicios de valor atribuidos a esta metodología, ya que cada dato utilizado en el proceso de determinación de la CCT fue elaborado con variables cuantificables y objetivas, eliminando las objeciones conceptuales de este proceso metodológico.

Al respecto, The Gale Encyclopedia of Science (2004) refiere que la capacidad de carga es un concepto muy útil porque señala el hecho de que hay un límite de población que puede ser sostenido, y aunque los humanos pueden desarrollar tecnologías para manipular la capacidad de carga en apoyo de actividades humanas, se tendrán que diseñar efectos compensatorios desde la economía humana, o los mismos ocurrirán naturalmente. 


\section{BIBLIOGRAFÍA}

- Administración Portuaria Integral de Quintana Roo API (2005). Puertos de Quintana Roo. Estadísticas.

Internet: http://www.apiqroo.com.mx/CGI-BIN/espanol/estadisticas.php

- BUTLER, Richard (1980). The concept of tourist area cycle of evolution: implications for management of resources. Canadian Geographer. XXIV. No. 1

- CAZES, George (1992). Fondements pour une Géographie du tourisme et des loisirs. Editorial Bréal, Paris, Francia.

- CIFUENTES, Miguel (1999). Capacidad de Carga Turística en las Áreas de uso público del Monumento Nacional Guayabo, Costa Rica.

Internet: www.wwfca.org/wwfpdfs/Guayabo.PDF (26/12/2006).

- COHEN, Eric (1972). Toward Sociology of International Tourism. Social Research.

- COOPER, Chris; FLETCHER, John; GILBERT, David; WANHILL, Steven (1997). Turismo Principio y Práctica. Editorial Diana. Ciudad de México, México.

- DATATUR - SECTUR (2007). Turismo en los Estados - Centro Turístico.

Internet: http://datatur.sectur.gob.mx/jsp/index.jsp

- DALY, Hernan \& COBB, John. Para el bien común. Reorientando la economía hacia la comunidad, el ambiente y un futuro sostenible. Editorial FCE, México, México.

- Enciclopedia Wikipedia WIKI (2007). Turismo sostenible.

Internet: http://es.wikipedia.org/wiki/Turismo_sostenible

- FFOLLIOTT, Meter; Bojorquez-Tapia, Luis; Hernandez-Narvaez, Mariano (2001). Natural Resources Management Practices. Editorial lowa State University Press. Ames - lowa, USA.

- Fundación para el Desarrollo Sustentable FDS (s.f.) Turismo Sustentable. Internet: http://www.fundacionsustentable.org/contentid-60.html (26/10/2007).

- GALE'S Encyclopedia of Science, 3rd. edition (2003). "Carrying Capacity". Digital Edition.

- GALLOPíN, Gilberto (2006). Los indicadores de desarrollo sostenible: Aspectos conceptuales y metodológicos.

Internet: $\quad$ www.rlc.fao.org/proyecto/fodepal/Bibvirtual/semex/indicadores/pdf/ gallopin.pdf (02/04/2007). 
- GARCÍA, María (2003). Turismo y conjuntos monumentales. Editorial Tirant Lo Blanch. Valencia, España

- Honorable Ayuntamiento de Cozumel HAC (2005). Plan de Desarrollo Municipal Cozumel 2005-2008 PDMC.

Internet: http://www.ordenjuridico.gob.mx/Estatal/QUINTANA\%20ROO/Municipios/ CozUmel/COZPLANO1.pdf (27/12/2006).

- Instituto Nacional de Estadística, Geografía e Informática INEGI (2007). Mapa Digital de México.

Internet: http://www.inegi.gob.mx/geo/default.aspx

http://galileo.inegi.gob.mx/website/mexico/default.htm? sistema $=1$ \&c $=423$ (15/06/2007).

- MALTHUS, Thomas (1998). Ensayo sobre el principio de población. Editorial FCE. México, México.

- MEADOWS; MEADOWS; RANDERS; BEHRENS, (1973). Los límites del crecimiento. Editorial FCE. México, México.

- MITRAUD, Sylvia (1997). Determinación de la Capacidad de Carga y Sistema de Monitoreo del Impacto de la Visitación. Proyecto de Desarrollo de Senderos Interpretativos del Parque Nacional Marino de Fernando de Noroña. Brasilia, Brasil.

- NARESTADO, J M. (2000). Sobre el origen, el uso y el contenido del término sostenible. Internet: http://habitat.aq.ump.es/cs/p2/a004.html (20/05/2007).

- NAVARRO, Enrique (2005). Metodología para la evaluación de la capacidad de carga turística. Malaga, España.

Internet: http://www.unia.es/nuevo_inf_academica/

visualizar_file_Adjunto.asp? $1 \mathrm{D}=1120 \quad(12 / 01 / 2007)$.

- Organización Mundial del Turismo OMT (2001). Turismo: Panorama 2020. Editorial OMT. Madrid, España.

- PED 2006-2011 (2006). Plan Estatal de Desarrollo 2006-2011. Documento final. Editorial Gobierno del Estado de Quintana Roo.

Internet: http://www.qroo.gob.mx/qroo/PlanGobierno/PlanEstatal.pdf. Quintana Roo, México. 
- PEDIEQRoo 2000-2025 (2000). Plan Estratégico de Desarrollo Integral del Estado de Quintana Roo 2000-2025. Reporte final. Editorial Centro de Estudios Estratégicos. Internet: http://www.xcalak.info/documents/Plan_estrategico_de_desarollo_intergal_ del_estado_de_qr_2000_2025-tomo1.pdf. Quintana Roo, México.

- Periódico Oficial del Gobierno del Estado de Quintana Roo POGEQR, publicado en fecha 21/05/2002. Decreto del Ordenamiento Ecológico Territorial de la Isla de Cozumel y su Área Marina de influencia.

Internet: $\quad$ http://www.semarnat.gob.mx/queessemarnat/ordenamientoecologico/ Documents/documentos\%20decretados/decreto_cozumel.pdf (11/01/2007).

- ROME, Abigail (1999). Monitoreo de los impactos turísticos: Un examen de metodologías y recomendaciones para el desarrollo de programas de monitoreo en América Latina. Internet: http://parksinperil.org/files/d_4_e_ecotourism_impacts_monitoring_spa.pdf (12/01/2007).

- SANTANA, Agustín (2003). Turismo cultural. Culturas turísticas.

Internet: www.scielo.br/pdf/ha/v9n20/v9n20a02.pdf (12/01/2007).

- Secretaría de Comunicaciones y Transporte SCT (2004). Anuarios estadísticos. Internet: http://e-mar.sct.gob.mx/index.php?id=520 (18/05/2007).

- Secretaria de Turismo SECTUR (2001). Programa Nacional de Turismo 2001-2006 PNT. Reporte final. Editorial SECTUR.

Internet: http://www.sectur.gob.mx/wb2/sectur/sect_767_capitulo_1. México, México

- Secretaría de Turismo del Estado de Quintana Roo SEDETUR (2007). Estadísticas. Indicadores Turísticos por año.

Internet: http://sedetur.qroo.gob.mx/index.php (30/05/2007).

- STAR, John; ESTES, Jeffry (1990). Geographic Information Systems: An introduction. Editorial Prentice Hall. New York, USA.

- WURZ, Jim (2003). Taller sobre el Monitoreo y Control de los Impactos del Turismo. Internet: www.conserveonline.org/docs/2003/07/TuristicoEspPt2.pdf 
APÉNDICE 1

Ficha técnica de indicador

CLAVE DEL INDICADOR: CZM-T06-0703

\section{NOMBRE: Superficie de espacio público por visitante}

Descripción del indicador: Es la superficie terrestre que utiliza una persona cualquiera para sus desplazamientos o movilidad natural. "El espacio público abarca, por regla general, las vías de circulación abiertas: calles, plazas, carreteras, parques, así como ciertos edificios públicos, como estaciones, bibliotecas públicas, ayuntamientos u otros. Los centros comerciales, por ejemplo, son espacios privados (en tanto que cierta gente, como los mendigos, tienen restringida la entrada) con apariencia de espacio público" (WIKI, 2007). Aquí también se considerarán las playas y otros sitios turísticos, como espacios públicos.

Objetivo del indicador: Medir la superficie del espacio público del cual dispone en promedio cada visitante.

Definición operativa: "Espacio público es el lugar donde cualquier persona tiene el derecho de circular, en oposición a los espacios privados, donde el paso puede ser restringido, generalmente por criterios de propiedad privada, reserva gubernamental u otros. Por tanto, espacio público es aquel espacio de propiedad pública y dominio y uso público". En este indicador si se considera a los centros comerciales como espacio público, ya que sólo se está considerando a los visitantes. Internet: http://es.wikipedia.org/wiki /Espacio_público (04/05/07).

Importancia: Este indicador es un complemento a otro indicador sobre Capacidad de Carga Turística.

Comentarios: Cifuentes (1999), en su estudio sobre capacidad de carga estima, o asume, que la superficie usada por persona $(\mathrm{sp})=1 \mathrm{~m}$. lineal de sendero por visitante. Este mismo criterio se presenta en el Municipio de Cozumel, en el Reglamento de Construcciones, en su capítulo II, 
Requerimientos de habitabilidad y funcionamiento, donde se menciona en el artículo 139 que los centros de reunión (espacios cerrados) deberán tener un mínimo de un metro cuadrado por persona, y en el artículo 164 se menciona que la capacidad de los templos se calculará un asistente por metro cuadrado y un volumen mínimo de $2.5 \mathrm{~m} 3$ por concurrente. No se mencionan criterios para espacios abiertos.

El estándar internacional de espacio público es de cuatro metros cuadrados por ciudadano (OMS, sf), de lo cual se deduce que el espacio lineal es de $2 \mathrm{~m}$., por lo que el factor de corrección estará dado por la diferencia entre la resultante de la fórmula y el estándar internacional. Asimismo, la OMT, en su guía práctica "Indicadores de desarrollo sostenible para los destinos turísticos" (2005) menciona que el espacio promedio necesario para realizar actividades recreativas es de " $4 \mathrm{~m} 2$ en espacios abiertos" (pag. 372).

Holder (1988), también hace referencia a parámetros de densidad óptima, tanto para destinos no explorados como para destinos maduros. En el primer caso, de oferta de playa virgen es recomendable establecer criterios de ocupación de $25 \mathrm{~m} 2 /$ usuario en áreas de reposo, pero en destinos turísticos maduros, con un sector turístico muy estacional, se limitará la superficie óptima máxima establecida a 15 m2/usuario en cada una de las playas estudiadas (Roig, 2000).

Por su parte, Aragonés y Amérigo (citado por García, 2003: 128 y 129) afirman que según la psicología ambiental, la distancia pública debe ser de $3.5 \mathrm{~m}$. a $7.25 \mathrm{~m}$. lineales entre personas, y el espacio mínimo, antes de considerarse hacinamiento, debería ser de $2 \mathrm{~m}$. lineales entre una persona y otra. En este documento se usará el criterio de $4 \mathrm{~m}$. lineales o $16 \mathrm{~m} 2$. para cada persona. 


\section{METODOLOGÍA}

Fuente de información: Directamente del Municipio local.

Periodicidad de la información: Anual.

\section{Calculo, variables /formula:}

SEPvis = Superficie de Espacio Público para Visitantes

Superficie de Espacio Público Efectivo / Promedio de visitantes por año

\begin{tabular}{|c|c|c|}
\hline Código & Unidad & Fórmula \\
\hline SEPvis & $\begin{array}{c}\text { metros cuadrados y lineales/ } \\
\text { visitante }\end{array}$ & SEPefe/VISita \\
\hline
\end{tabular}

Variables necesarias para construir el indicador

\begin{tabular}{|l|l|l|}
\hline \multicolumn{1}{|c|}{ Nombre } & \multicolumn{1}{|c|}{ Unidad } & \multicolumn{1}{c|}{ Descripción } \\
\hline SEPefe & Metro cuadrado y lineal & $\begin{array}{l}\text { Superficie de espacio público } \\
\text { efectivo }\end{array}$ \\
\hline VISita & Visitantes & Número de visitantes al municipio \\
\hline
\end{tabular}

\section{FACTOR DE CORRECCIÓN}

$\mathrm{FCsup}=1-\frac{\mathrm{x}}{16}$

Donde x es la magnitud limitante, o sea el resultado de una resta: (16 - SEPvis).

Si SEPvis es superior al estándar internacional, no existe factor de corrección. 


\title{
CITAS
}

\begin{abstract}
1 Aunque los términos "sostenible" y "sustentable" pueden conceptualizarse como sinónimos en el uso cotidiano y no parece justificable diferenciarlos (GALLOPÍN, 2006), en este documento se utilizará la palabra "sustentable", ya que la Ley General del Equilibrio Ecológico y Protección del Ambiente -LGEEPA-, artículo III, fracción XI (1997) utiliza dicha palabra.

2 En México, es común que se clasifique a los visitantes en turistas extranjeros y turistas nacionales, debido a la disponibilidad de información y a las diferencias en el gasto turístico, ya que se atribuye un gasto turístico muy superior a los turistas extranjeros, aunque aquí se debe considerar la excepción, ya que los cruceristas y excursionistas (transbordador) si bien son de origen extranjero, tiene un gasto turístico muy limitado -US\$ 538 para turistas de hotel, contrastado con los US\$35 para excursionistas o cruceristas- , y al considerar exclusivamente a los cruceristas, el promedio de gasto es de US\$8.2 por persona. (SEDETUR Quintana Roo, 2004: Internet).
\end{abstract}

${ }^{3}$ La Capacidad de Carga Turística es un concepto derivado de la administración de recursos, y la Organización Mundial del Turismo OMT (citado por CAZES, 1992) la define como el número máximo de personas que pueden visitar un destino turístico al mismo tiempo, sin causar destrucción del medio físico, económico o sociocultural, y una disminución inaceptable en la satisfacción de los visitantes, aunque se considera conveniente excluir de esta definición la frase "satisfacción de los visitantes" debido a que se orienta hacia el lado de la demanda mientras que los demás factores considerados corresponden al lado de la oferta, y el objetivo principal de la CCT es proteger el atractivo turístico, principalmente del uso turístico.

4 El concepto de "estrategia" es fundamental en el análisis de la capacidad de carga turística, ya que la CCT no determina la fragilidad de los ecosistemas locales. Para ello es necesario realizar estudios de resistencia y resilencia.

5 Sobre este punto y la derrama económica vinculada, existen estudios que indican que la industria de cruceros ocupa el segundo lugar, mientras que el primer lugar lo tiene el turismo de buceo, ya que en los informes oficiales no queda clara la diferencia entre ingresos turísticos y derrama económica.

${ }^{6}$ Este argumento no pretende hacer una apología de la propiedad privada, sino presentar un hecho social concreto -aunque no exclusivo- de Cozumel: las personas cuidan más lo privado que lo público, para maximizar las ganancias individuales y repartir los costos a nivel social. Igualmente, esta situación se repite en la actividad turística, con el agregado de que los efectos se multiplican debido a las personas adicionales que debe soportar 
el destino turístico y que no forman parte de la sociedad local. Como resultados de este comportamiento, el valor del bien público disminuye para todos y luego se agota.

7 En este documento se hace la diferencia conceptual entre "destino turístico" y "sitio turístico", ya que un destino puede poseer varios sitios turísticos.

${ }^{8}$ Con respecto a la herramienta, el GPS (Global Position System, por sus siglas en inglés) debe ser ajustado al Sistema de proyección en UTM (Universal Tranversal Marcador) con el criterio Map Datum WGS84, que es utilizado para el SIG en México, con las unidades en metros.

9 Se debe hacer notar que con este método no es necesario determinar la cantidad de visitantes o la presión turística que recibe el destino o sitio turístico. Sin embargo, una vez establecida la CCT deberán monitorearse todos sus indicadores componentes, como procedimiento normal de regulación de la CCT. 


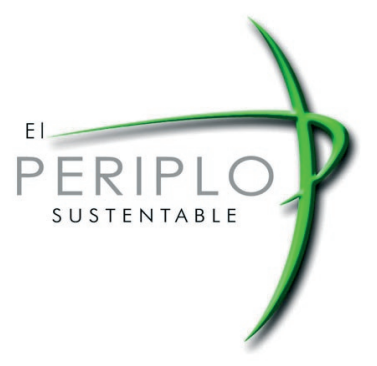

FICHA BIBLIOGRÁFICA:

SEGRADO, Romano; PALAFOX, Alejandro; ARROYO, Lucinda.

Medición de la Capacidad de Carga Turística de Cozumel.

El Periplo Sustentable. Espacio de análisis y reflexión sobre Turismo Sustentable. México: Universidad Autónoma del Estado de México, enero de 2008, núm. 13

<http://www.uaemex.mx/plin/psus/rev13/articulo_02.pdfs.

[ISSN: 1870-9036]. 